\title{
A Risk-Adjusted Evaluation Of The JSE Top 40 As An International Investment Option
}

André Heymans, North-West University, South Africa

Chris van Heerden, North-West University, South Africa

\begin{abstract}
In response to the wealth destruction caused by the 2007/2008 global financial crisis, many developed economies have lowered their interest rates to improve their balance sheets (SARB, 2008-2012). However, in order for investors to sustain expected returns they will have to deviate from the traditional approach of investing in government bonds and consider investing in emerging markets, which are considered as potential drivers of global growth (Deloitte Consulting LLP, 2012). The goal of this paper is to establish the importance of considering South Africa as an emerging market investment opportunity, but also to acknowledge its ability of outperforming several other common emerging markets during the post-financial crisis period. This was done by means of a novel approach to the Omega ratio. The results from this paper confirms this, illustrating that the performance of the JSE Top 40 will compensate for the additional political risk that emerging market investments possess (Anshuman, 2010).
\end{abstract}

Keywords: Emerging Markets; Omega Ratio; Risk-Adjusted Performance; JSE

\section{INTRODUCTION}

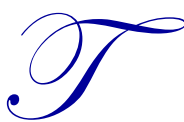

here is a large body of literature on the returns characteristics of emerging markets. Many academics points out that investing in emerging markets are beneficial in international portfolios because of their low correlation with the rest of the developed world (Claessens, Dasgupta \& Glen, 1995; Rouwenhorst, 1999), and because emerging market returns seem to be influenced more by local rather than global information variables. This naturally serves as a good diversification for international portfolios. Various studies also points out that expected returns in emerging markets are higher than those in developed economies (Lesmond, 2005), and that these returns are more predictable than returns in the developed world (Harvey, 1995).

However, despite the possibility of earning higher returns, many also warn that emerging market investments expose investors to various risks. There seems to be widespread consensus on what these risks are. Bekaert and Harvey (1997) for example note that emerging markets have more predictable returns and higher volatility than developed markets. This theme is also repeated by De Santis and Imrohoroğlu (1997) and Bekaert and Harvey (2002) to name but a few. However, as these economies become more open, their markets become less volatile (Bekaert \& Harvey, 1997). It is this reduction in volatility, low correlation to the rest of the developed world, as well as the opportunity to share in the higher returns that made emerging markets a popular investment destination over the past two decades. Between 1991 and 2000 alone the total value of stocks traded increased from $\$ 15$ billion to $\$ 200$ billion, whilst market capitalization increased from $\$ 306$ billion to more than $\$ 1.4$ trillion (Lesmond, 2005).

African markets too have become popular amongst international investors of late. Many international investors view Africa as the final investment frontier. This continent houses not only an untapped market in terms of consumers, but investors too find value in Africa. In 1995 African stock exchanges gained about 40 per cent and by 2004 average returns on African stock exchanges were still doing well at 44 per cent (Alagidede \& Panagiotidis, 
2009). These returns have been spurred on by the trend amongst African countries to liberalise their markets while encouraging privatisation of government firms, implementing automated trading systems and launching educational programs to attract investors (Irving, 2005). It is also noteworthy that some of the bigger economies in Africa fall within the emerging markets paradigm. At the economic head of Africa sits South Africa, the biggest economy in Africa. South Africa also boasts the most liquid stock market and has the biggest market capitalisation in Africa (Smith \& Dyakova, 2013).

South Africa, as many other developing economies, has been doing well on the back of the economic growth of the developed world in the past decade. However, as emerging economies became more integrated over the last 25 years, they became more susceptible to financial crises (Mendoza \& Smith, 2013). The financial crisis of 2007/08 was no exception. This crisis, which initially manifested in the US real estate market (Kamin \& DeMarco, 2012), led to the weakening of both consumer and investor confidence worldwide (SARB, 2008). With the weakened consumer demand and business sentiment, increased inflationary pressures tightened funding conditions in financial markets. This caused deterioration in global manufacturing activities that led to the fall of world economic growth and contributed to the greater risk associated with obtaining global financial stability (SARB, 2008). The consequences of the financial crisis also made insurable profitable investment decisions extremely difficult as market volatility tends to increase during crisis periods. This was again visible with the 2007/08 crisis leading to a re-coupling of developed and emerging markets (Mun \& Brooks, 2013).

Nonetheless, during the post-2007/08 financial crisis there was a significant trend among investors to acquire financial assets in Emerging Market Economies (EMEs). As a result, the equity markets in the EMEs were able to outperform developed markets with gains of 61 per cent by the end of September 2009 (SARB, 2009). This increase in confidence in EME equities was partly due to the stimulus of their fiscal policy (SARB, 2009) and the lower interest rates in advanced economics (SARB, 2010). However, investors started to discard these equities again in 2011, and EMEs were adversely influenced because of uncertain US and European sovereign debt markets (SARB, 2011). This risk aversion against EMEs' equities continued in 2012, and investors' preference towards equities in advanced economies escalated (SARB, 2012). This was because the slowdown in advanced economies spilled over to the EMEs, with the fear that the slowdown in China's economic growth may hold significant financial and economic stability implications (SARB, 2012). However, optimism arose regarding the attractiveness for EME equities in 2013, when manufacturing and service-sector activities increased, along with greater confidence in China's seeming success in overcoming their economic growth slowdown (SARB, 2013).

Despite the fact that investment decisions during the late crisis and post-crisis periods were mainly based on the performance between advanced and emerging markets, as highlighted above, investors did not always recognise the ability of developing economies to provide secure investments due to the contagion effect from the US economy to the rest of the world. This is particularly true for the South African economy, where uncertainty surrounds its classification as a developing economy or a possible emerging market, which have been considered as one of the top performers among the large emerging equity markets (SARB, 2012). To this end, Table 1 compares the South African JSE All Share and JSE Top 40 indices with the most common advanced and emerging markets. 
Table 1: Performance Comparison Between Markets - January 2010 To December 2013

\begin{tabular}{|c|c|c|c|c|c|c|c|c|}
\hline Name & $\begin{array}{l}\text { Minimum } \\
\text { Returns }\end{array}$ & $\begin{array}{l}\text { Maximum } \\
\text { Returns }\end{array}$ & \multicolumn{2}{|c|}{$\begin{array}{l}\text { Average } \\
\text { Returns }\end{array}$} & \multicolumn{2}{|c|}{$\begin{array}{l}\text { Cumulative } \\
\text { returns }\end{array}$} & $\begin{array}{l}\text { Standard } \\
\text { Deviation }\end{array}$ & $\begin{array}{l}\text { Risk-adjusted } \\
\text { returns }\end{array}$ \\
\hline Dow Jones & $-5.546 \%$ & $4.241 \%$ & \multicolumn{2}{|c|}{$0.051 \%$} & \multicolumn{2}{|c|}{$12.311 \%$} & 0.027 & $1.859 \%$ \\
\hline CAC 40 & $-5.479 \%$ & $9.659 \%$ & \multicolumn{2}{|c|}{$0.019 \%$} & \multicolumn{2}{|c|}{$2.170 \%$} & 0.046 & $0.407 \%$ \\
\hline DAX & $-5.819 \%$ & $5.349 \%$ & \multicolumn{2}{|c|}{$0.055 \%$} & \multicolumn{2}{|c|}{$12.283 \%$} & 0.051 & $1.067 \%$ \\
\hline S\&P 500 & $-6.663 \%$ & $4.741 \%$ & \multicolumn{2}{|c|}{$0.056 \%$} & \multicolumn{2}{|c|}{$13.495 \%$} & 0.029 & $1.915 \%$ \\
\hline JSE Top 40 & $-3.763 \%$ & $4.577 \%$ & \multicolumn{2}{|c|}{$0.057 \%$} & \multicolumn{2}{|c|}{$13.615 \%$} & 0.028 & $2.001 \%$ \\
\hline JSE All Share & $-3.627 \%$ & $4.324 \%$ & \multicolumn{2}{|c|}{$0.057 \%$} & \multicolumn{2}{|c|}{$13.828 \%$} & 0.026 & $2.196 \%$ \\
\hline Mexican IPC index & $-5.810 \%$ & $4.255 \%$ & \multicolumn{2}{|c|}{$0.033 \%$} & \multicolumn{2}{|c|}{$7.402 \%$} & 0.028 & $1.179 \%$ \\
\hline FTSE 100 index & $-4.667 \%$ & $5.161 \%$ & \multicolumn{2}{|c|}{$0.027 \%$} & \multicolumn{2}{|c|}{$5.665 \%$} & 0.030 & $0.904 \%$ \\
\hline S\&P BSE Sensex & $-4.125 \%$ & $3.773 \%$ & \multicolumn{2}{|c|}{$0.025 \%$} & \multicolumn{2}{|c|}{$5.015 \%$} & 0.039 & $0.645 \%$ \\
\hline Nikkei 225 index & $-10.554 \%$ & $5.678 \%$ & \multicolumn{2}{|c|}{$0.054 \%$} & \multicolumn{2}{|c|}{$11.767 \%$} & 0.053 & $1.011 \%$ \\
\hline SC index ${ }^{\&}$ & $-5.299 \%$ & $4.324 \%$ & \multicolumn{2}{|c|}{$-0.037 \%$} & \multicolumn{2}{|c|}{$-10.538 \%$} & 0.039 & $-0.943 \%$ \\
\hline \multicolumn{9}{|c|}{ RANKINGS PER CATEGORY FROM BEST TO WORST } \\
\hline $\begin{array}{c}\text { Minimum } \\
\text { Returns }\end{array}$ & $\begin{array}{l}\text { Maximum } \\
\text { Returns }\end{array}$ & \multicolumn{2}{|c|}{ Average Returns } & \multicolumn{2}{|c|}{$\begin{array}{l}\text { Cumulative } \\
\text { Returns }\end{array}$} & \multicolumn{2}{|c|}{$\begin{array}{l}\text { Standard } \\
\text { Deviation } \\
\end{array}$} & $\begin{array}{l}\text { Risk-adjusted } \\
\text { returns }\end{array}$ \\
\hline JSE All Share & CAC 40 & \multicolumn{2}{|c|}{ JSE Top 40} & \multicolumn{2}{|c|}{ JSE All Share } & \multicolumn{2}{|c|}{ JSE All Share } & JSE All Share \\
\hline JSE Top 40 & Nikkei 225 index & \multicolumn{2}{|c|}{ JSE All Share } & & op 40 & & Jones & JSE Top 40 \\
\hline S\&P BSE Sensex & DAX & $\mathrm{S} \& \mathrm{P}$ & & & 500 & & $\begin{array}{l}\text { can IPC } \\
\text { ldex }\end{array}$ & S\&P 500 \\
\hline FTSE 100 index & FTSE 100 index & $\mathrm{DA}$ & & & ones & & Top 40 & Dow Jones \\
\hline SC index \& & S\&P 500 & Nikkei 2 & & & & & P 500 & $\begin{array}{l}\text { Mexican IPC } \\
\text { index }\end{array}$ \\
\hline CAC 40 & JSE Top 40 & Dow & & Nikk & 25 index & & 100 index & DAX \\
\hline Dow Jones & JSE All Share & $\begin{array}{r}\text { Mexice } \\
\text { ind } \\
\end{array}$ & & & $\begin{array}{l}\text { n IPC } \\
\text { ex }\end{array}$ & & index \& & Nikkei 225 index \\
\hline $\begin{array}{l}\text { Mexican IPC } \\
\text { index }\end{array}$ & SC index \& & FTSE 10 & & FTS & 0 index & & SE Sensex & FTSE 100 index \\
\hline DAX & $\begin{array}{c}\text { Mexican IPC } \\
\text { index }\end{array}$ & S\&P BSF & & $\mathrm{S} \& \mathrm{P}$ & Sensex & & $\mathrm{AC} 40$ & S\&P BSE Sensex \\
\hline S\&P 500 & Dow Jones & $\mathrm{CAC}$ & & & & & $\mathrm{AX}$ & CAC 40 \\
\hline Nikkei 225 index & S\&P BSE Sensex & $\mathrm{SC}$ in & & & $\operatorname{dex}^{\&}$ & & 225 index & SC index ${ }^{\&}$ \\
\hline
\end{tabular}

\# Note that this table reports the annualised standard deviation to provide a more comprehensive risk perception.

* Cumulative returns were estimated based on a 252 trading day horizon.

${ }^{+}$Risk-adjusted returns were estimated by dividing the average returns with the annualised standard deviation.

$\&$ SC index $=$ Shanghai Composite index

Source: Data were obtained from Yahoo Finance (2014) and McGregor (2014), respectively.

From Table 1 it is evident that both the JSE All Share and JSE Top 40 indices performed relatively well against the other markets. It displayed little fluctuations in terms of the minimum and maximum returns and the JSE All Share and the JSE Top 40 indices also ranked first and fourth with the lowest standard deviation, respectively, from a total of 11 markets. Furthermore, the JSE All Share and JSE Top 40 indices exhibited significant consistency in terms of average and cumulative returns, where it ranked under the top three markets, respectively (see Table 1). From these results the conclusion can be drawn that the JSE All Share and JSE Top 40 indices rank under the top three in terms of risk-adjusted returns, making it a suitable investment option to consider during the post-financial crisis period along with other investment options that advanced and emerging markets offer. Furthermore, due to the fact that equities are a preferred asset class to protect the purchasing power of investors in an environment of high inflation (Alagidede \& Panagiotidis, 2010), it becomes imperative to evaluate the risk-adjusted performance of the South African equity market to justify its validity as an investment option. This leads to the purpose of this paper, which is twofold; to evaluate the more common South African index, namely the JSE Top 40, with the Omega ratio to highlight the significance of specific South African shares, and to do so by improving on the current form of the Omega ratio itself. The rest of this paper consists of an overview on performance measures and the methodology in Section 2, followed by a discussion of the data and the results in Sections 3 and 4, respectively. Section 5 will then conclude with the conclusion and recommendations. 


\section{PERFORMANCE MEASURES AND METHODOLOGY}

Although the Sharpe ratio (1966) remains one of the most commonly used statistics in financial analysis (see for example Schuster and Auer, 2012; Auer and Schuhmacher, 2013) it also has some limitations. One of the biggest limitations of the Sharpe ratio is that it makes use of the standard deviation as a risk measure. Although the standard deviation proves sufficient in some instances it can easily be manipulated by seeking returns in "nonnormal risks", like extreme liquidity, credit risk and volatility variation risks (Amenc, Martellini \& Sfeir, 2004:2). Another important limitation is that it does not differentiate between upside risk and downside risk, thus also penalising positive returns (De Wet, Krige \& Smit, 2008). Thirdly, the Sharpe ratio operates independently of any fund benchmark in estimating excess returns, making the evaluation of some portfolios difficult (Amenc, Martellini $\&$ Sfeir, 2004). Fourthly, the Sharpe ratio also assumes that the returns of the individual security are uncorrelated with the mean portfolio returns; a process that might lead to misleading performance rankings in the process (Sharpe, 1994). Finally, the Sharpe ratio also operates on the assumption that returns are normally distributed. This is seldom the case, especially when working with emerging market returns (Hwang \& Pedersen, 2004). This will limit the Sharpe ratio's performance ranking abilities (Amin \& Kat, 2003), especially when accounting for the increased divergence from normality occurring in the higher moments of the return distributions (Kat, 2003). Furthermore, different portfolio allocations will be possible with the presence of non-normal returns, when comparing the traditional mean-variance framework, developed by Markowitz (1952), to more advanced performance measures, like discussed below (see for example Fung \& Hsieh, 1999a; Cvitanić, Lazrak, Martellini \& Zapatero, 2003; Lamm, 2003; Terhaar, Staub \& Singer, 2003; Popova, Morton \& Popova, 2003; and Wong, Phoon \& Lean, 2008).

Additionally, several approaches have been developed to overcome some of the Sharpe ratio's weaknesses. The following ratios were for example developed as a substitute, which include the modified Sharpe ratio (Gregoriou \& Gueyie, 2003); the modified Value at Risk (MVaR) model (Favre \& Galeano, 2002); the Conditional Drawdown at Risk (CDaR) model; the Conditional Value at Risk (CVaR) model (Krokhmal, Palmquist \& Uryasev, 2002); the Cornish-fisher ratio (Liang \& Park, 2007); as well as the Polynomial Goal Programming process (PGP) used by Davies, Kat and Lu (2009). The paper by Salomons and Grootveld (2003) also made use of lower partial moment (LPM) models, by means of the Sortino ratio, to model emerging market returns. Another paper attempted to applied the Capital Asset Pricing Model (CAPM), but found that it was unable to capture the risk-return relationship of emerging market returns (Hwang \& Pedersen, 2004). This failure was addressed by applying a LPMCAPM, although it still failed to improve the results of the CAPM model (Hwang \& Pedersen, 2004). Nonetheless, most of the above mentioned models suffer from the inability to capture all the risk-return characteristics in a return distribution. In order to overcome this shortcoming, this paper will apply an Omega ratio, as introduced by Keating and Shadwick (2002), to model emerging market returns. The Omega ratio, as a risk-adjusted performance ratio, still emphasise the importance of the risk of loss (Pedersen, Rudhulm-Alfvin, 2003), but treats upside and downside risk differently, thus "heeding" the criticism of the mean-variance portfolio optimisation of Markowitz (1952) (Gilli, Schumann, Di Tollo \& Cabej, 2011:95). The Omega ratio also includes all the information that are encoded in all the moments (variance, mean, skewness, and kurtosis) without any prior assumptions (De Wet, Krige \& Smit, 2008).

The Omega ratio is, therefore, beneficial as it considers both the upside potential (higher partial moments) and downside potential (lower partial moments) of an investment over the entire distribution. This differs from other ratios such as the Sharpe and Sortino ratios (see Sortino \& Price, 1994), as well as the Calmar ratio (see Young, 1991), which only considers the lower partial moments (downside risk and maximum drawdown, respectively). The Omega ratio can, therefore, be formulated as follows (Eling \& Schuhmacher, 2007):

$\Omega(r)=\frac{\int_{r}^{b}(1-F(x)) d x}{\int_{a}^{r} F(x) d x}$,

where $r$ denotes the selected threshold; $x$ denotes the random one-period return of an investment; $a$ and $b$ denote the upper and lower bounds of the return distribution, respectively; $\int_{r}^{b}(1-F(x)) d x$ denotes the upside potential; and $\int_{a}^{r} F(x) d x$ denotes the downside potential. Although the Omega ratio overcomes a great number of inefficiencies 
that plague other performance measures, it is still fallible in that it is backward-looking. Also, it can discriminate between different underlying fund strategies and between different periods of various types of market activities (Botha, 2007).

To ensure that the Omega ratio will provide desirable results it is important to apply a suitable threshold. As such, a risk-free rate is recommended by several studies; see for example Botha (2007), Eling and Schuhmacher (2007) and de Wet, Krige and Smit (2008). However, this approach has two weaknesses, which will lead to inconsistent performance rankings. Firstly, the threshold can change depending on an investor's performance preferences, which implies that different rankings will be possible for the same portfolio. Secondly, some investors consider minimising downside risk more important that maximising profit and vice versa, which can also lead to different Omega rankings. To overcome these weaknesses an alternative approach was implemented, which considers estimating the trend (slope) of the entire Omega function. This implies that two individual Ordinary-Least Squared (OLS) regressions were estimated, one for each side (positive \& negative) of the distribution. A share will, therefore, be more desirable if it has a steep slope on the negative side $\left(\mathrm{S}_{2}\right)$ of the distribution and a flat slope on the positive side $\left(S_{1}\right)$ of the distribution. This implies that the share with the smallest ratio $\left(S_{1} \div S_{2}\right)$ will be more desirable in terms of performance. However, the two slopes must be estimated within an area of the distribution where the different Omega functions of each asset can still be distinguished, and before each function reaches infinity. After estimating the Omega function for each asset, this area was determined between -2.25 and +2.25 , as illustrated in Figure 1.

\section{DATA}

This paper employs the daily closing prices of the JSE Top 40 shares, which is based on the market capitalisation as on 30 July 2013, when this paper commenced (see Table 2). In order to substantiate the performance of the JSE Top 40 shares, this paper will also make use of the daily index values of the JSE All Share, the JSE Top 40 and several world indices (see Table 3). The data were obtained from the McGregor BFA (2014) database and from Yahoo Finance (2014), respectively, spanning from January 2010 to December 2013.

The choice of this time frame is to only capture the effects of the post-financial crisis on the performance of the JSE Top 40 shares. Due to a lack of data the Capital \& Countries Properties Plc, the Rand Merchant Insurance Holdings Limited, the NBKIOEXXSTUB10, the NBNPNR268.50CII and the Life Healthcare Group Holdings Limited share prices were excluded from the list of JSE Top 40 shares under investigation. 
Figure 1: Omega As A Function Of Returns - Introducing A New Threshold Approach

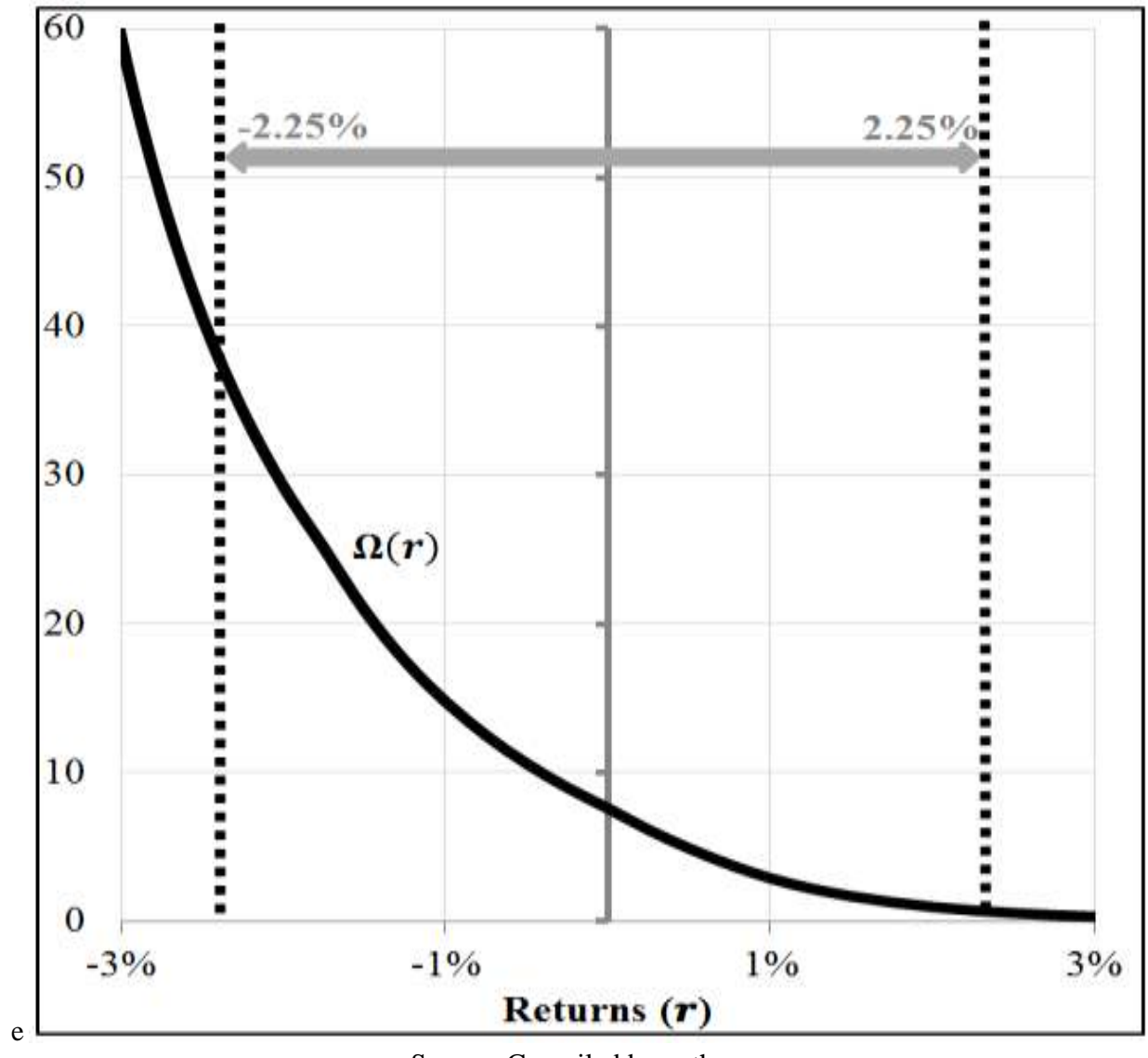

Source: Compiled by authors. 
Table 2: The JSE Top 40 Shares - According To Highest Market Capitalisation At 30 July 2013

\begin{tabular}{|c|c|c|c|c|c|}
\hline Ticker & Name & Sector & Industry & Sub-industry & $\begin{array}{c}\text { Market } \\
\text { Capitalisation (Rand) }\end{array}$ \\
\hline BTI & $\begin{array}{l}\text { British American } \\
\text { Tobacco Plc }\end{array}$ & Consumer stables & Consumer products & Tobacco & 1055781382590 \\
\hline SAB & SABMiller Plc & Consumer staples & Consumer products & Beverages & 787559096007 \\
\hline $\mathrm{BIL}$ & BHP Billiton Plc & Materials & Iron \& steel & $\begin{array}{c}\text { Steel raw material } \\
\text { suppliers }\end{array}$ & 610051841953 \\
\hline CFR & $\begin{array}{c}\text { Compagnie Financière } \\
\text { Richemont }\end{array}$ & $\begin{array}{c}\text { Consumer } \\
\text { discretionary } \\
\end{array}$ & $\begin{array}{l}\text { Apparel \& textile } \\
\text { products }\end{array}$ & $\begin{array}{c}\text { Apparel, footwear, } \\
\text { accessories }\end{array}$ & 484624800000 \\
\hline NPN & Naspers Limited & Communications & Media & Local media & 333904872477 \\
\hline MTN & MTN Group Limited & Communications & Telecom & Telecom carriers & 331595120510 \\
\hline AGL & Anglo American Plc & Materials & Metals \& mining & Base metals & 302441463754 \\
\hline SOL & Sasol Limited & Energy & Oil, gas \& coal & Integrated oils & 292478522259 \\
\hline SBK & $\begin{array}{c}\text { Standard Bank Group } \\
\text { Limited }\end{array}$ & Financials & Banking & Banks & 177165273588 \\
\hline VOD & $\begin{array}{c}\text { Vodacom Group } \\
\text { Limited }\end{array}$ & Communications & Telecom & Telecom carriers & 173063929740 \\
\hline FSR & FirstRand Limited & Financials & Banking & Banks & 164909794403 \\
\hline OML & Old Mutual Plc & Financials & Insurance & Life insurance & 141504708341 \\
\hline $\mathrm{KIO}$ & $\begin{array}{c}\text { Kumba Iron Ore } \\
\text { Limited }\end{array}$ & Materials & Iron \& steel & $\begin{array}{c}\text { Steel raw material } \\
\text { suppliers }\end{array}$ & 141292675074 \\
\hline SLM & Sanlam Limited & Financials & Insurance & Life insurance & 98553000000 \\
\hline APN & $\begin{array}{c}\text { Aspen Pharmacare } \\
\text { Holdings } \\
\end{array}$ & Health care & $\begin{array}{c}\text { Biotech \& } \\
\text { pharmaceuticals }\end{array}$ & $\begin{array}{c}\text { Generic } \\
\text { Pharmaceuticals }\end{array}$ & 97209082840 \\
\hline AMS & $\begin{array}{c}\text { Anglo American } \\
\text { Platinum Corporation } \\
\text { Limited }\end{array}$ & Materials & Metals \& mining & $\begin{array}{l}\text { Precious metal } \\
\text { mining }\end{array}$ & 94118978214 \\
\hline SHP & $\begin{array}{c}\text { Shoprite Holdings } \\
\text { Limited } \\
\end{array}$ & Consumer staples & Retail staples & Food retailers & 93460915548 \\
\hline NED & $\begin{array}{c}\text { Nedbank Group } \\
\text { Limited }\end{array}$ & Financials & Banking & Banks & 90265358378 \\
\hline REM & Remgro Limited & Consumer staples & Consumer products & $\begin{array}{c}\text { Food } \\
\text { manufacturing }\end{array}$ & 88975812067 \\
\hline BVT & $\begin{array}{c}\text { The Bidvest Group } \\
\text { Limited }\end{array}$ & Consumer staples & $\begin{array}{l}\text { Distribute/Wholesal } \\
\text { e consumer staples }\end{array}$ & $\begin{array}{c}\text { Food product } \\
\text { wholesalers }\end{array}$ & 79147985353 \\
\hline IMP & $\begin{array}{l}\text { Impala Platinum } \\
\text { Holdings Limited }\end{array}$ & Materials & Metals \& mining & $\begin{array}{l}\text { Precious metal } \\
\text { mining }\end{array}$ & 63221427600 \\
\hline TBS & Tiger Brands Limited & Consumer staples & Consumer products & $\begin{array}{c}\text { Food } \\
\text { manufacturing }\end{array}$ & 59660806282 \\
\hline $\mathrm{RMH}$ & $\begin{array}{l}\text { RMB Holdings } \\
\text { Limited }\end{array}$ & Financials & Speciality finance & $\begin{array}{c}\text { Other financial } \\
\text { services }\end{array}$ & 56355192462 \\
\hline MDC & $\begin{array}{c}\text { Mediclinic } \\
\text { International } \\
\end{array}$ & Health care & $\begin{array}{c}\text { Health care } \\
\text { facilities/services }\end{array}$ & $\begin{array}{c}\text { Health care } \\
\text { services }\end{array}$ & 56224828526 \\
\hline WHL & $\begin{array}{c}\text { Woolworths Holdings } \\
\text { Limited }\end{array}$ & Consumer staples & Retail staples & Food retailers & 55403811768 \\
\hline EXX & $\begin{array}{c}\text { Exxaro Resources } \\
\text { Limited }\end{array}$ & Energy & Oil, gas \& coal & Coal operations & 55223779647 \\
\hline DSY & Discovery Limited & Financials & Insurance & Life insurance & 53002172524 \\
\hline ANG & $\begin{array}{c}\text { AngloGold Ashanti } \\
\text { Limited }\end{array}$ & Materials & Metals \& mining & $\begin{array}{l}\text { Precious metal } \\
\text { mining }\end{array}$ & 51077414689 \\
\hline MNP & Mondi Plc & Materials & $\begin{array}{c}\text { Containers \& } \\
\text { packaging }\end{array}$ & $\begin{array}{l}\text { Containers \& } \\
\text { packaging } \\
\text { manufacturing }\end{array}$ & 50873868716 \\
\hline
\end{tabular}


(Table 2 continued)

\begin{tabular}{|c|c|c|c|c|c|}
\hline ITU & Intu Properties Plc & Financials & $\begin{array}{c}\text { Real estate } \\
\text { investment trust }\end{array}$ & $\begin{array}{l}\text { Retail real estate } \\
\text { investment trust }\end{array}$ & 48543131731 \\
\hline SHF & $\begin{array}{l}\text { Steinhoff International } \\
\text { Holdings Limited }\end{array}$ & $\begin{array}{c}\text { Consumer } \\
\text { discretionary }\end{array}$ & $\begin{array}{l}\text { Home \& office } \\
\text { products }\end{array}$ & $\begin{array}{l}\text { Home \& office } \\
\text { furnishings }\end{array}$ & 48290855354 \\
\hline GRT & $\begin{array}{c}\text { Growthpoint } \\
\text { Properties Limited }\end{array}$ & Financials & $\begin{array}{c}\text { Real estate } \\
\text { operations \& } \\
\text { services }\end{array}$ & $\begin{array}{c}\text { Office owners \& } \\
\text { developers }\end{array}$ & 47421367282 \\
\hline ASR & Assore Limited & Materials & Iron \& steel & $\begin{array}{c}\text { Steel raw material } \\
\text { suppliers }\end{array}$ & 47117362500 \\
\hline GFI & Gold Fields Limited & Materials & Metals \& mining & $\begin{array}{c}\text { Precious metal } \\
\text { mining }\end{array}$ & 43884903263 \\
\hline IPL & $\begin{array}{c}\text { Imperial HoldingS } \\
\text { Limited }\end{array}$ & $\begin{array}{c}\text { Consumer } \\
\text { discretionary }\end{array}$ & Retail discretionary & Auto retail stores & 42810911575 \\
\hline INP & Investec Plc & Financials & Banking & Banks & 40278615070 \\
\hline TRU & $\begin{array}{c}\text { Truworths } \\
\text { International Limited }\end{array}$ & $\begin{array}{c}\text { Consumer } \\
\text { discretionary }\end{array}$ & Retail discretionary & $\begin{array}{c}\text { Speciality apparel } \\
\text { stores }\end{array}$ & 38022881791 \\
\hline REI & $\begin{array}{l}\text { Reinet Investments } \\
\text { S.C.A. }\end{array}$ & Financials & Speciality finance & $\begin{array}{c}\text { Other financial } \\
\text { services }\end{array}$ & 37130873697 \\
\hline MSM & $\begin{array}{l}\text { Massmart Holdings } \\
\text { Limited }\end{array}$ & Consumer staples & Retail staples & Mass merchants & 36026355232 \\
\hline ARI & $\begin{array}{c}\text { African Rainbow } \\
\text { Minerals }\end{array}$ & Materials & Iron \& steel & $\begin{array}{c}\text { Steel raw material } \\
\text { suppliers }\end{array}$ & 35707495363 \\
\hline
\end{tabular}

Source: Compiled by authors and the fundamental information were collected from the Bloomberg (2014) database.

Table 3: List of indices under investigation

\begin{tabular}{|c|c|c|}
\hline Name & Origin & Classification \\
\hline Dow Jones & North America & Advanced market \\
\hline S\&P 500 & North America & Advanced market \\
\hline CAC 40 & France & Advanced market \\
\hline DAX & Germany & Advanced market \\
\hline FTSE 100 & United Kingdom & Emerging market \\
\hline S\&P BSE Sensex & India & Emerging market \\
\hline SC index & China & Advanced market \\
\hline Nikkei 225 index & Japan & Emerging market \\
\hline Mexican IPC index & South America (Latin America) & Emerging market \\
\hline JSE All Share index & South Africa & Emerging market \\
\hline JSE Top 40 index & South Africa & \\
\hline
\end{tabular}

Source: Compiled by authors.

\section{RESULTS}

The first step of the empirical study is to establish the descriptive statistics of the time series under investigation. The results reported in Table 4 exhibits a clear indication that all the JSE Top 40 shares and indices are leptokurtic, with a kurtosis greater than three. These findings emphasise the results found by Fung and Hsieh (1999b), who argued that return series are known to be leptokurtic. Also, half of the JSE Top 40 shares exhibits a negative skewness, whereas all the indices except the CAC 40 and the S\&P BSE Sensex Bombay Index illustrate a negative skewness. This implies that investors which invest in these shares or markets may be exposed to a downside surprise (see for example McFall Lamm, 2003). Furthermore, it can be argued that variance, standard deviation and beta will not be able to provide a perception of the actual risk involved, where these measures will only demonstrate how the positive returns will be penalised (Kat, 2003). 
Table 4: Descriptive Statistics Of The JSE Top 40 Shares And World Indices

\begin{tabular}{|c|c|c|c|c|c|c|c|c|c|}
\hline Name & Mean & Median & Max. & Min. & Skewness & Kurtosis & $\begin{array}{c}\text { Jarque- } \\
\text { Bera }\end{array}$ & $\begin{array}{c}\text { Anderson- } \\
\text { Darling }\end{array}$ & $\begin{array}{c}\text { Shapiro- } \\
\text { Wilk }\end{array}$ \\
\hline AGL & 0.000 & 0.000 & 0.078 & -0.058 & 0.242 & 3.673 & 28.686 & 0.820 & 0.994 \\
\hline AMS & 0.000 & -0.001 & 0.083 & -0.077 & 0.274 & 4.015 & 55.418 & 2.229 & 0.990 \\
\hline ANG & -0.001 & -0.001 & 0.119 & -0.077 & 0.515 & 6.266 & 488.589 & 5.319 & 0.965 \\
\hline APN & 0.001 & 0.001 & 0.081 & -0.057 & 0.306 & 4.683 & 133.596 & 4.440 & 0.981 \\
\hline ARI & 0.000 & 0.000 & 0.088 & -0.072 & 0.184 & 4.006 & 47.841 & 2.146 & 0.991 \\
\hline ASR & 0.001 & 0.000 & 0.202 & -0.085 & 0.814 & 10.599 & 2516.745 & 14.617 & 0.935 \\
\hline BIL & 0.000 & 0.000 & 0.062 & -0.052 & 0.191 & 3.661 & 24.330 & 1.025 & 0.994 \\
\hline BTI & 0.001 & 0.001 & 0.044 & -0.043 & -0.113 & 3.905 & 36.266 & 1.252 & 0.993 \\
\hline BVT & 0.001 & 0.000 & 0.071 & -0.052 & 0.180 & 4.818 & 143.184 & 4.638 & 0.979 \\
\hline CFR & 0.002 & 0.001 & 0.081 & -0.072 & 0.092 & 4.814 & 138.573 & 4.690 & 0.980 \\
\hline DSY & 0.001 & 0.001 & 0.066 & -0.091 & -0.345 & 7.480 & 856.047 & 6.679 & 0.961 \\
\hline EXX & 0.001 & 0.001 & 0.065 & -0.071 & -0.077 & 3.387 & 7.223 & 1.482 & 0.996 \\
\hline FSR & 0.001 & 0.001 & 0.059 & -0.106 & -0.291 & 5.015 & 183.258 & 0.978 & 0.987 \\
\hline GFI & -0.001 & -0.001 & 0.134 & -0.135 & 0.154 & 6.889 & 634.307 & 4.499 & 0.966 \\
\hline GRT & 0.001 & 0.000 & 0.068 & -0.055 & -0.066 & 6.074 & 394.496 & 9.810 & 0.960 \\
\hline IMP & 0.000 & 0.000 & 0.092 & -0.062 & 0.231 & 3.688 & 28.557 & 1.832 & 0.992 \\
\hline INP & 0.001 & 0.001 & 0.085 & -0.073 & -0.075 & 4.753 & 128.925 & 2.721 & 0.985 \\
\hline IPL & 0.001 & 0.000 & 0.067 & -0.054 & 0.170 & 3.520 & 16.090 & 1.497 & 0.995 \\
\hline ITU & 0.000 & 0.000 & 0.079 & -0.206 & -2.224 & 34.647 & 42554.090 & 9.760 & 0.880 \\
\hline KIO & 0.001 & 0.000 & 0.078 & -0.076 & -0.008 & 4.357 & 76.788 & 4.491 & 0.983 \\
\hline MDC & 0.001 & 0.000 & 0.069 & -0.071 & 0.006 & 6.155 & 414.847 & 8.460 & 0.963 \\
\hline MNP & 0.002 & 0.002 & 0.075 & -0.082 & 0.118 & 4.735 & 127.750 & 4.259 & 0.982 \\
\hline MSM & 0.000 & 0.000 & 0.106 & -0.080 & 0.274 & 6.811 & 617.535 & 4.329 & 0.970 \\
\hline MTN & 0.001 & 0.001 & 0.082 & -0.074 & -0.017 & 3.902 & 33.947 & $0.744 *$ & 0.994 \\
\hline NED & 0.001 & 0.001 & 0.060 & -0.061 & 0.052 & 4.226 & 63.091 & 1.336 & 0.990 \\
\hline NPN & 0.001 & 0.002 & 0.082 & -0.065 & -0.044 & 4.043 & 45.623 & 3.297 & 0.989 \\
\hline OML & 0.001 & 0.001 & 0.117 & -0.159 & -0.606 & 16.417 & 7561.785 & 5.791 & 0.923 \\
\hline REI & 0.001 & 0.000 & 0.050 & -0.034 & 0.123 & 4.000 & 44.197 & 2.960 & 0.990 \\
\hline REM & 0.001 & 0.001 & 0.064 & -0.045 & 0.147 & 4.283 & 72.188 & 2.223 & 0.988 \\
\hline RMH & 0.001 & 0.001 & 0.071 & -0.305 & -3.920 & 65.499 & 165317.900 & 8.769 & 0.832 \\
\hline SAB & 0.001 & 0.002 & 0.058 & -0.067 & -0.131 & 4.502 & 96.875 & 2.445 & 0.988 \\
\hline SBK & 0.000 & 0.000 & 0.051 & -0.063 & -0.118 & 4.171 & 59.457 & 1.543 & 0.990 \\
\hline SHF & 0.001 & 0.000 & 0.053 & -0.053 & 0.124 & 3.451 & 11.036 & 1.674 & 0.995 \\
\hline SHP & 0.001 & 0.001 & 0.058 & -0.059 & -0.059 & 3.611 & 16.155 & $0.684^{*}$ & 0.996 \\
\hline SLM & 0.001 & 0.001 & 0.057 & -0.051 & -0.012 & 4.559 & 101.236 & 5.670 & 0.980 \\
\hline SOL & 0.001 & 0.001 & 0.056 & -0.045 & 0.029 & 3.538 & 12.217 & 0.939 & 0.996 \\
\hline TBS & 0.001 & 0.001 & 0.059 & -0.061 & -0.145 & 3.760 & 27.529 & 1.064 & 0.995 \\
\hline
\end{tabular}




(Table 4 continued)
\begin{tabular}{|c|c|c|c|c|c|c|c|c|c|}
\hline TRU & 0.001 & 0.001 & 0.065 & -0.081 & -0.329 & 4.236 & 81.702 & 1.587 & 0.988 \\
\hline VOD & 0.001 & 0.001 & 0.056 & -0.084 & -0.141 & 4.976 & 165.957 & 2.344 & 0.984 \\
\hline WHL & 0.002 & 0.001 & 0.086 & -0.058 & -0.013 & 4.064 & 47.221 & 1.702 & 0.991 \\
\hline JSE Top 40 & 0.001 & 0.001 & 0.046 & -0.038 & -0.104 & 4.214 & 63.166 & 3.450 & 0.986 \\
\hline JSE All Shares & 0.001 & 0.001 & 0.043 & -0.036 & -0.155 & 4.281 & 72.349 & 3.478 & 0.986 \\
\hline Dow Jones & 0.001 & 0.001 & 0.042 & -0.055 & -0.351 & 6.800 & 625.976 & 13.184 & 0.947 \\
\hline CAC 40 & 0.000 & 0.000 & 0.097 & -0.055 & 0.150 & 6.643 & 571.180 & 7.581 & 0.962 \\
\hline DAX & 0.001 & 0.001 & 0.053 & -0.058 & -0.089 & 5.600 & 290.509 & 11.059 & 0.961 \\
\hline S\&P 500 & 0.001 & 0.001 & 0.047 & -0.067 & -0.373 & 7.140 & 741.766 & 13.906 & 0.944 \\
\hline Mexican IPC index & 0.000 & 0.000 & 0.043 & -0.058 & -0.356 & 5.747 & 337.814 & 5.909 & 0.972 \\
\hline FTSE 100 index & 0.000 & 0.000 & 0.052 & -0.047 & -0.108 & 5.127 & 192.099 & 6.223 & 0.975 \\
\hline S\&P BSE Sensex Bombay & 0.000 & 0.000 & 0.038 & -0.041 & 0.042 & 3.681 & 19.432 & 1.873 & 0.994 \\
\hline Nikkei 225 index & 0.001 & 0.001 & 0.057 & -0.106 & -0.720 & 7.751 & 1011.779 & 2.695 & 0.962 \\
\hline SC index & 0.000 & 0.000 & 0.043 & -0.053 & -0.223 & 4.798 & 141.540 & 6.094 & 0.977 \\
\hline
\end{tabular}

* Implies that the null hypothesis for a normal distribution is rejected at a $10 \%$ confidence interval.

To further emphasise these arguments, three normality tests are reported in order to justify the presence of non-normality. The first normality test entails the Jarque-Bera test (Jarque \& Bera, 1987), which is dependent on higher moments (skewness \& kurtosis) to differentiate between types of distributions. Although, the skewness and kurtosis coefficient have several disadvantages that have to be acknowledged with the evaluation of the return distribution characteristics. Firstly, both have an unbounded influence function and both have zero breakdown value, which imply that bias estimates could be generated with the presence of outliers. Secondly, both are only defined on distributions that have finite moments (Brys, Hubert \& Struyf, 2008). Nonetheless, evidence has been found which illustrated that the Jarque-Bera test gives the most powerful results for normal distributions (Öztuna, Elhan \& Tüccar, 2006). To provide more convincing results however, two additional normality tests will be implemented which are based on two different foundations. This includes the test introduced by Anderson and Darling (1952), which is based on an empirical distribution function, where its applicability has also been justified by several studies, including Thadewald and Büning (2007) as well as Balakrishnan, Chimitova, Galanova and Vedernikova (2013).

The third normality test is that of Shapiro \& Wilk (1965), which is based on correlation. The superiority of the Shapiro-Wilk test over the AndersonDarling and the Jarque-Bera test has been confirmed by Bradley and Morris (2013). However, Yap and Sim (2011) found that the Jarque-Bera test will illustrate similar performance with symmetric long-tailed distributions, whereas the Anderson-Darling and the Shapiro-Wilk tests tend to be more powerful normality tests with the presence of asymmetric distributions.

The presence of a normal distribution is rejected at a five per cent confidence interval for all the JSE Top 40 shares and for all the indices, except for Shoprite Holdings Limited (SHP) and for MTN Group Limited (MTN), which rejected the null hypothesis of normality at a ten per cent confidence interval. These findings accentuate the inability of traditional risk-adjusted performance measures, (which make use of variance, standard deviation or beta to generate a reliable performance ranking; see for example Bernardo \& Ledoit, 2000; Lamm, 2003) to rank investment portfolios. This justifies the importance of using the Omega ratio instead.

In addition, the second step of the empirical study is to evaluate the risk-adjusted performance of the JSE Top 40 shares by means of the Omega ratio. From the results obtained from the Omega ratio, as reported in Table 5, it is further substantiated that there are South African investment options that have the 
ability to outperform most common world indices. Note that only the top 20 rankings will be discussed, whereas the complete Omega rakings are available in Table $\mathrm{A}$ in the appendix. The results reported that the metals and mining industry $\left(1^{\text {st }} \& 3^{\text {rd }}\right.$ place $)$ and the iron and steel industry $\left(2^{\text {nd }}\right.$ place $)$ were overall the three best performing sectors, respectively, over the period under investigation. Individually, it was Impala Platinum Holding Limited (IMP), Assore Limited (ASR) and Anglo American Platinum Corporation Limited (AMS) that were the top three performing shares. It is interesting to note that there was no correlation between the Omega rankings, the actual Top 40 rankings (based on market capitalisation), the average closing price, the average trading volume or the average Price-to-Earnings (P/E) ratio. Though, from the 16 shares that were able to outperform all the world indices, (with the CAC 40 ranking the highest) only eight shares exhibited an average P/E ratio lower than that of the JSE All Share index (15.441) and of the JSE Top 40 index (15.789). These findings, therefore, suggest that there are seven shares which are undervalued and should be considered as more promising investment options compared to the other shares, whereas Intu Properties Plc (ITU) exhibited a negative average P/E ratio and will be excluded from further discussions. These seven shares include Exxaro Resources Limited (EXX), Kumba Iron Ore Limted (KIO), BHP Billiton Plc. (BIL), Assore Limited (ASR), FirstRand Limited (FSR), Standard Bank Group (SBK) and Investec Plc (INP), respectively. Furthermore, Remgro Limited (REM), Discovery Limited (DSY) and Tiger Brands Limited (TBS) were the three poorest performing shares over the period under investigation (see Table A in the appendix), respectively, making consumer products and insurance the less desirable South African industries.

Table 5: Top 20 Performance Ranking Based On The Omega Ratio - Ranked From Best To Worst

\begin{tabular}{|c|c|c|c|c|c|c|c|}
\hline Ticker & Name & Industry & $\begin{array}{c}\text { Market } \\
\text { Capitalisation (Rand) } \\
\end{array}$ & $\begin{array}{l}\text { JSE Top } 40 \text { Market } \\
\text { Cap ranking }\end{array}$ & $\begin{array}{l}\text { Ave. Price } \\
\text { (in cent) }\end{array}$ & Ave. Volume & Ave. P/E \\
\hline IMP & Impala Platinum Holdings Limited & Metals \& mining & 63221427600 & 22 & 16365.668 & 2279765.126 & 29.220 \\
\hline ASR & Assore Limited & Iron \& steel & 47117362500.00 & 34 & 25637.893 & 74558.390 & 12.609 \\
\hline AMS & $\begin{array}{l}\text { Anglo American Platinum } \\
\text { Corporation Limited }\end{array}$ & Metals \& mining & 94118978214.00 & 17 & 54744.475 & 367652.765 & 42.915 \\
\hline ARI & African Rainbow Minerals & Iron \& steel & 35707495363 & 1 & 18297.654 & 443369.964 & 21.139 \\
\hline MNP & Mondi Plc & Containers \& packaging & 50873868716 & 30 & 8189.448 & 910740.367 & 24.034 \\
\hline ITU & Intu Properties Plc & Real estate investment trust & 48543131731 & 31 & 4503.445 & 1113958.112 & -8.935 \\
\hline EXX & Exxaro Resources Limited & Oil, gas \& coal & 55223779647 & 27 & 15667.477 & 978295.021 & 11.462 \\
\hline INP & Investec Plc & Banking & 40278615070 & 37 & 5554.914 & 1574844.578 & 13.748 \\
\hline AGL & Anglo American Plc & Metals \& mining & 302441463754.00 & 8 & 28478.241 & 3943631.195 & 19.797 \\
\hline KIO & Kumba Iron Ore Limited & Iron \& steel & 141292675074 & 14 & 46057.598 & 483641.348 & 11.679 \\
\hline RMH & RMB Holdings Limited & Speciality finance & 56355192462 & 24 & 3481.790 & 2050052.888 & 2369.179 \\
\hline NPN & Naspers Limited & Media & 333904872477 & 6 & 47900.352 & 1687722.747 & 38.109 \\
\hline TRU & Truworths International Limited & Retail discretionary & 38022881791 & 38 & 7745.994 & 1637317.629 & 16.684 \\
\hline SBK & Standard Bank Group Limited & Banking & 177165273588 & 10 & 10838.166 & 4066060.335 & 12.757 \\
\hline FSR & FirstRand Limited & Banking & 164909794403 & 12 & 2434.614 & 12716436.582 & 12.663 \\
\hline APN & Aspen Pharmacare Holdings & Biotech \& pharmaceuticals & 97209082840.00 & 16 & 12937.751 & 1207954.462 & 22.184 \\
\hline BIL & BHP Billiton Plc & Iron \& steel & 610051841953.00 & 4 & 25775.196 & 3309468.520 & 12.001 \\
\hline \multicolumn{8}{|c|}{ CAC 40} \\
\hline CFR & Compagnie Financière Richemont & Apparel \& textile products & 484624800000 & 5 & 5263.588 & 9046622.864 & 22.361 \\
\hline SAB & SABMiller Plc & Consumer products & 787559096007 & 3 & 32474.891 & 1668051.606 & 25.137 \\
\hline
\end{tabular}

${ }^{+}$Note: This table only reports the top 20 rankings, whereas the complete Omega ratio ranking is available in the Appendix.

Source: The Fundamental data for the JSE shares and indices were also collected from the McGregor BFA (2014) database. 
Moreover, from a world index perspective it was the CAC 40 and the Shanghai Composite index who performed the best, whereas the S\&P BSE Sensex index and the Mexican IPC index illustrated the poorest riskadjusted performance (see Table A in the appendix). Although, the JSE All Share index failed to perform well relative to the other world indices $\left(3^{\text {rd }}\right.$ poorest), the risk-adjusted performance of several individual JSE Top 40 shares emphasised the possibility that South Africa, as a developing market, may have the ability to contribute more to an international investment portfolio compared to some advanced and emerging markets. This proves that the South African equity market exhibited more of the performance characteristics of an emerging market compared to the general expectations of the ability of a developing market.

\section{CONCLUSION}

Investing in emerging markets is considered to be beneficial to international portfolios, as it provides a lower correlation with the rest of the developed world and higher expected returns compared to other markets. These features were especially beneficial to investors during the post-crisis period, where there was a significant increase in the demand for financial assets in emerging markets. However, it was not long until the effects of the financial crisis spilled over to these emerging markets, causing doubt and encouraging investors to return to investing in developed markets. This paper however proves that the South African equity market, more specifically the JSE Top 40 shares, have more promise to an international investor compared to what most common world indices have to offer. The findings illustrate that there were 16 JSE Top 40 shares that were able to outperform most common world indices, with Impala Platinum Holding Limited (IMP), Assore Limited (ASR) and Anglo American Platinum Corporation Limited (AMS) being the top performing shares, respectively. It was also interesting to note from these 16 shares it was only seven shares who reported a lower average P/E ratio compared to the JSE All Share index and the JSE Top 40 index. These shares include Exxaro Resources Limited (EXX), Kumba Iron Ore Limted (KIO), BHP Billiton Plc. (BIL), Assore Limited (ASR), FirstRand Limited (FSR), Standard Bank Group (SBK) and Investec Plc (INP), respectively.

\section{REFERENCES}

1. Alagidede, P., \& Panagiotidis, T. (2009). Modelling stock returns in Africa's emerging equity markets. International review of financial analysis, 18(1) 1-11.

2. Alagidede, P., \& Panagiotidis, T. (2010). Can common stocks provide a hedge against inflation? Evidence from African countries. Review of financial economics, 19(3) 91-100.

3. Amenc, N., Martellini, L., \& Sfeir, D. (2004). Methodology applied for the AGEFI asset management awards. Edhec risk and asset management research centre. 18p.

4. Amin, G.S., \& Kat, H.M. (2003). Hedge fund performance 1990-2000: Do the money machines really add value? Journal of financial and quantitative analysis, 38(2) 251-274.

5. Anderson, T.W., \& Darling, D.A. (1952). Asymptotic theory of certain "goodness of fit" criteria based on stochastic processes. The annuals of mathematical statics, 23(2) 193-212.

6. Anshuman, V.R. (2010). Sovereign risk and investing in emerging markets. [Web]: http://ssrn.com/abstract=1685442. [Date of access]: 27 February 2014.

7. Auer, B.R. and Schuhmacher, F. (2013). Robust evidence on the similarity of Sharpe ratio and drawdownbased hedge fund performance rankings. Journal of international financial markets, institutions and money, 24(1) 153-165.

8. Balakrishnan, N., Chimitova, E., Galanova, N., \& Vedernikova, M. (2013). Testing the Goodness of fit of parametric AFT and PH models with residuals. Communications in statistics - simulation and computation, $42(6)$ 1352-1367.

9. Bekaert, G., \& Harvey, C.R. (1997). Emerging equity market volatility. Journal of financial economics, 43(1) 29-77.

10. Bekaert, G., \& Harvey, C.R. (2002). Research in emerging markets finance: looking to the future. Emerging markets review, 3(4) 429-448.

11. Bernardo, A.E., \& Ledoit, O. (2000). Gain, loss and asset pricing. Journal of political economy, 108(1) 144-172.

12. Botha, M. (2007). A comparison of South African hedge fund risk measures. South African journal of economics, 75(3) 459-477. 
13. Bradley, D., \& Morris, J.M. (2013). On the performance of negentropy approximations as test statistics for detecting sinusoidal RFI in microwave radiometers. IEEE transactions on geoscience and remote sensing, 51(10) 4945-4951.

14. Brys, G., Hubert, M., \& Struyf, A. (2008). Goodness-of-fit tests based on a robust measure of skewness. Computational statistics, 23(3) 429-442.

15. Claessens, S., Dasgupta, S., \& Glen, J. (1995). Return behavior in emerging stock markets. World bank economic review, 9(1) 131-151.

16. Cvitanić, J., Lazrak, A., Martellini, L., \& Zapatero, F. (2003). Optimal allocation to hedge funds: An empirical analysis. Quantitative finance, 3(1) 28-39.

17. Davies, R.J., Kat, H.M., \& Lu, S. (2009). Fund of hedge funds portfolio selection: A multiple objective approach. Journal of derivatives and hedge funds, 15(2) 91-115.

18. Deloitte Consulting LLP. (2012). Fortresses and footholds: Emerging market growth strategies, practices and outlook. [Web]: https://www.deloitte.com/assets/Dcom-UnitedStates/Local\%20

19. Assets/Documents/us_consulting_Fortresses\%20and\%20Footholds_111511.pdf. [Date of access]: 27 February 2014.

20. De Santis, G., \& Imrohoroğlu, S. (1997). Stock returns and volatility in emerging financial markets. Journal of international money and finance, 16(4) 561-579.

21. De Wet, R., Krige, J.D., \& Smit, E. vd M. (2008). An investigation into performance rankings of the Omega ratio vs. the Sharpe ratio applied to South African general equity unit trusts. Journal of studies in economics and econometrics, 32(2) 69-83.

22. Eling, M., \& Schuhmacher, F. (2007). Does the choice of performance measure influence the evaluation of hedge funds? Journal of banking and finance, 31(9) 2632-2647.

23. Favre, L., \& Galeano, J.A. (2002). Mean-Modified Value at Risk with hedge funds. The journal of alternative investments, 5(2) 21-25.

24. Fung, W., \& Hsieh, D.A. (1999a). Is mean-variance analysis applicable to hedge funds? Economic letters, 62(1) 53-58.

25. Fung, W., \& Hsieh, D.A. (1999b). A primer on hedge funds. Journal of empirical finance, 6(3) 309-331.

26. Gilli, M., Schumann, E., Di Tollo, G., \& Cabej, G. (2011). Constructing 130/30-portfolios with the Omega ratio. Journal of asset management, 12(2) 94-108.

27. Gregoriou, G.N., \& Gueyie, J-P. (2003). Risk-adjusted performance of funds of hedge funds using a Modified Sharpe ratio. Journal of wealth management, 6(3) 77-83.

28. Harvey, C.R. (1995). The risk exposure of emerging equity markets. The world bank economic review, 9(1) 19-50.

29. Hwang, S., \& Pedersen, C.S. (2004). Asymmetric risk measures when modelling emerging markets equities: Evidence for regional and timing effects. Emerging markets review, 5(1) 109-128.

30. Irving, J. (2005). Regional integration of stock exchanges in Eastern and Southern Africa: Progress and prospects. IMF Working Paper, WP/05/122. [Web]: http://www.iadb.org/intal/intalcdi/pe/2010/

31. 06075.pdf. [Date of access]: 11 February 2014.

32. Jarque, C.M., \& Bera, A.K. (1987). A test for normality of observations and regression residuals. International statistical review, 55(2) 163-172.

33. Kamin, S.B., \& DeMarco, L.P. (2012). How did a domestic housing slump turn into a global financial crisis? Journal of international money and finance, 31(1) 10-41.

34. Kat, H.M. (2003). 10 Things investors should know about hedge funds. The Journal of wealth management, 5(4) 72-81.

35. Keating, C., \& Shadwick, W.F. (2002). A universal performance measure. Journal of performance measurement, 6(3) 59-84.

36. Krokhmal, P., Palmquist, J., \& Uryasev, S. (2002). Portfolio optimization with conditional Value-at-Risk objective and constraints. Journal of risk, 4(2) 43-68.

37. Lamm, M.R. (2003). Asymmetric returns and optimal hedge fund portfolios. Journal of alternative investments, 6(2) 9-21.

38. Lesmond, D.A. (2005). Liquidity of emerging markets. Journal of financial economics, 77(2) 411-452.

39. Liang, B., \& Park, H. (2007). Risk measures for hedge funds: A cross-sectional approach. European financial management, 13(2) 333-370.

40. Markowitz, H. (1952). Portfolio selection. Journal of finance, 7(1) 77-91. 
41. McFall Lamm, R. (2003). Asymmetric returns and optimal hedge fund portfolios. Journal of Alternative Investments, 6(2) 9-21.

42. McGregor BFA database. (2014). Supplier of data. [Web]: https://expert.mcgregorbfa.com/. [Date of access]: 5 February 2014.

43. Mendoza, E.G., \& Smith, K.A. (2013). Financial globalization, financial crises, and the external portfolio structure of emerging markets. The Scandinavian journal of economics, 116(1) 20-57.

44. Mun, M., \& Brooks, R. (2013). The roles of news and volatility in stock market correlations during the global financial crisis. Emerging markets review, 13(1) 1-7.

45. Ö̈ztuna, D., Elhan, A.H., \& Tüccar, E. (2006). Investigation of four different normality tests in terms of type 1 error rate and power under different distributions. Turkish journal of medical science, 36(3) 171176.

46. Pedersen, C.S., \& Rudholm-Alfvin, T. (2003). Selecting a risk-adjusted shareholder performance measure. Journal of asset management, 4(3) 152-172.

47. Popova, I., Morton, D.P., \& Popova, E. (2003). Optimal hedge fund allocation with asymmetric preferences and distributions. [Website]: http://www.me.utexas.edu/ popova/

48. OptHedgeFundAllocation.pdf. [Date of access]: 11 February 2014.

49. Rouwenhorst, K.G. (1999). Local return factors and turnover in emerging stock markets. The journal of finance, 54(4) 1439-1464.

50. Salomons, R., \& Grootveld, H. (2003). The equity risk premium: Emerging vs. developed markets. Emerging markets review, 4(2) 121-144.

51. SARB (South African Reserve Bank). (2008). Financial stability review. September 2008. South African Reserve Bank. 45p.

52. SARB (South African Reserve Bank). (2009). Financial stability review. September 2009. South African Reserve Bank. 45p.

53. SARB (South African Reserve Bank). (2010). Financial stability review. September 2010. South African Reserve Bank. 38p.

54. SARB (South African Reserve Bank). (2011). Financial stability review. September 2011. South African Reserve Bank. 52p.

55. SARB (South African Reserve Bank). (2012). Financial stability review September 2012. South African Reserve Bank. 57p.

56. SARB (South African Reserve Bank). (2013). Financial stability review March 2013. South African Reserve Bank. 62p.

57. Schuster, M. and Auer, B.R. (2012). A note on empirical Sharpe ratio dynamics. Economics letters, 116(1) 124-128.

58. Shapiro, S.S., \& Wilk, M.B. (1965). An analysis of variance test for normality (complete samples). Biometrika, 52(3/4) 591-611.

59. $\quad$ Sharpe, W.F. (1994). The Sharpe ratio. Journal of portfolio management, 21(1) 49-58.

60. Sharpe, W.F. (1966). Mutual fund performance. Journal of business, 39(1) 119-138.

61. Smith, G., \& Dyakova, A. (2013). African stock markets: Efficiency and relative predictability. South African journal of economics, doi: 10.1111/saje.12009

62. Sortiono, F.A., \& Prince, L. (1994). Performance measurement in a downside risk framework. Journal of investing, 3(3) 59-64.

63. Terhaar, K., Staub, R., \& Singer, B. (2003). Appropriate policy allocation for alternative investments: A factor approach and simulation techniques. Journal of portfolio management, 29(3) 101-110.

64. Thadewald, T., \& Büning, H. (2007). Jarque-Bera test and its competitors for testing normality - A power comparison. Journal of applied statistics, 34(1) 87-105.

65. Wong, W-K., Phoon, K. F., \& Lean, H.H. (2008). Stochastic dominance analysis of Asian hedge funds. Pacific-Basin finance journal, 16(3) 204-223.

66. Yahoo Finance. (2014). Supplier of data. [Web]: http://finance.yahoo.com/. [Date of access]: 5 February 2014.

67. Yap, B.W., \& Sim, C.H. (2011). Comparison of various types of normality tests. Journal of statistical computation and simulations, 81(12) 2141-2155.

68. Young, T.W. (1991). Calmar ratio: A smoother tool. Futures, 20(1) 40. 


\section{APPENDIX}

Table A: Complete Omega Ranking - Ranked From Best To Worst

\begin{tabular}{|c|c|c|c|c|c|}
\hline $\begin{array}{c}\text { Omega } \\
\text { Ranking }\end{array}$ & Ticker & Name & Industry & $\begin{array}{c}\text { Market } \\
\text { Capitalisation (Rand) }\end{array}$ & $\begin{array}{c}\text { JSE Top 40 } \\
\text { Market Cap Ranking }\end{array}$ \\
\hline 1 & IMP & $\begin{array}{l}\text { Impala Platinum } \\
\text { Holdings Limited }\end{array}$ & Metals \& mining & 63221427600 & 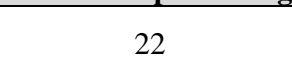 \\
\hline 2 & ASR & Assore Limited & Iron \& steel & 47117362500.00 & 34 \\
\hline 3 & AMS & $\begin{array}{c}\text { Anglo American } \\
\text { Platinum Corporation } \\
\text { Limited } \\
\end{array}$ & Metals \& mining & 94118978214.00 & 17 \\
\hline 4 & ARI & $\begin{array}{c}\text { African Rainbow } \\
\text { Minerals } \\
\end{array}$ & Iron \& steel & 35707495363 & 1 \\
\hline 5 & MNP & Mondi Plc & $\begin{array}{c}\text { Containers \& } \\
\text { packaging }\end{array}$ & 50873868716 & 30 \\
\hline 6 & ITU & Intu Properties Plc & $\begin{array}{c}\text { Real estate } \\
\text { investment trust }\end{array}$ & 48543131731 & 31 \\
\hline 7 & EXX & $\begin{array}{c}\text { Exxaro Resources } \\
\text { Limited }\end{array}$ & Oil, gas \& coal & 55223779647 & 27 \\
\hline 8 & INP & Investec Plc & Banking & 40278615070 & 37 \\
\hline 9 & AGL & Anglo American Plc & Metals \& mining & 302441463754.00 & 8 \\
\hline 10 & $\mathrm{KIO}$ & $\begin{array}{c}\text { Kumba Iron Ore } \\
\text { Limited }\end{array}$ & Iron \& steel & 141292675074 & 14 \\
\hline 11 & $\mathrm{RMH}$ & $\begin{array}{l}\text { RMB Holdings } \\
\text { Limited }\end{array}$ & Speciality finance & 56355192462 & 24 \\
\hline 12 & NPN & Naspers Limited & Media & 333904872477 & 6 \\
\hline 13 & TRU & $\begin{array}{c}\text { Truworths } \\
\text { International Limited }\end{array}$ & Retail discretionary & 38022881791 & 38 \\
\hline 14 & SBK & $\begin{array}{c}\text { Standard Bank Group } \\
\text { Limited }\end{array}$ & Banking & 177165273588 & 10 \\
\hline 15 & FSR & Firstrand Limited & Banking & 164909794403 & 12 \\
\hline 16 & APN & $\begin{array}{c}\text { Aspen Pharmacare } \\
\text { Holdings }\end{array}$ & $\begin{array}{c}\text { Biotech \& } \\
\text { pharmaceuticals }\end{array}$ & 97209082840.00 & 16 \\
\hline 17 & $\mathrm{BIL}$ & BHP Billiton Plc & Iron \& steel & 610051841953.00 & 4 \\
\hline 18 & \multicolumn{5}{|c|}{ CAC 40} \\
\hline 19 & CFR & $\begin{array}{c}\text { Compagnie Financière } \\
\text { Richemont }\end{array}$ & $\begin{array}{l}\text { Apparel \& textile } \\
\text { products }\end{array}$ & 484624800000 & 5 \\
\hline 20 & SAB & SABMiller Plc & Consumer products & 787559096007 & 3 \\
\hline 21 & OML & Old Mutual Plc & Insurance & 141504708341 & 13 \\
\hline 22 & MSM & $\begin{array}{c}\text { Massmart Holdings } \\
\text { Limited }\end{array}$ & Retail staples & 36026355232 & 40 \\
\hline 23 & WHL & $\begin{array}{c}\text { Woolworths Holdings } \\
\text { Limited }\end{array}$ & Retail staples & 55403811768 & 26 \\
\hline 24 & GFI & Gold Fields Limited & Metals \& mining & 43884903263 & 35 \\
\hline 25 & MTN & MTN Group Limited & Telecom & 331595120510 & 7 \\
\hline 26 & NED & $\begin{array}{c}\text { Nedbank Group } \\
\text { Limited }\end{array}$ & Banking & 90265358378 & 19 \\
\hline 27 & MDC & $\begin{array}{c}\text { Mediclinic } \\
\text { International }\end{array}$ & $\begin{array}{c}\text { Health care } \\
\text { facilities/services }\end{array}$ & 56224828526 & 25 \\
\hline 28 & IPL & $\begin{array}{c}\text { Imperial HoldingS } \\
\text { Limited }\end{array}$ & Retail discretionary & 42810911575 & 36 \\
\hline
\end{tabular}


(Table A continued)

\begin{tabular}{|c|c|c|c|c|c|}
\hline 29 & \multicolumn{5}{|c|}{ Shanghai Composite index } \\
\hline 30 & SHF & $\begin{array}{c}\text { Steinhoff International } \\
\text { Holdings Limited }\end{array}$ & $\begin{array}{l}\text { Home \& office } \\
\text { products }\end{array}$ & 48290855354 & 32 \\
\hline 31 & ANG & $\begin{array}{l}\text { AngloGold Ashanti } \\
\text { Limited }\end{array}$ & Metals \& mining & 51077414689.00 & 29 \\
\hline 32 & SLM & Sanlam Limited & Insurance & 98553000000 & 15 \\
\hline 33 & \multicolumn{5}{|c|}{$\begin{array}{r}\text { S\&P 500 } \\
\end{array}$} \\
\hline 34 & BVT & $\begin{array}{c}\text { The Bidvest Group } \\
\text { Limited }\end{array}$ & $\begin{array}{c}\text { Distribute/Wholesale } \\
\text { consumer staples }\end{array}$ & 79147985353.00 & 21 \\
\hline 35 & VOD & $\begin{array}{c}\text { Vodacom Group } \\
\text { Limited } \\
\end{array}$ & Telecom & 173063929740 & 11 \\
\hline 36 & \multicolumn{5}{|c|}{ Nikkei 225 index } \\
\hline 37 & SHP & $\begin{array}{c}\text { Shoprite Holdings } \\
\text { Limited }\end{array}$ & Retail staples & 93460915548 & 18 \\
\hline 38 & \multicolumn{5}{|c|}{ JSE Top 40 index } \\
\hline 39 & SOL & Sasol Limited & Oil, gas \& coal & 292478522259 & 9 \\
\hline 40 & \multicolumn{5}{|c|}{ DAX } \\
\hline 41 & \multicolumn{5}{|c|}{ Dow Jones } \\
\hline 42 & BTI & $\begin{array}{l}\text { British American } \\
\text { Tobacco Plc } \\
\end{array}$ & Consumer products & 1055781382590.00 & 2 \\
\hline 43 & \multicolumn{5}{|c|}{ FTSE 100 index } \\
\hline 44 & \multicolumn{5}{|c|}{$\begin{array}{l}\text { JSE All Share index } \\
\end{array}$} \\
\hline 45 & GRT & $\begin{array}{c}\text { Growthpoint Properties } \\
\text { Limited }\end{array}$ & $\begin{array}{c}\text { Real estate operations } \\
\text { \& services }\end{array}$ & 47421367282 & 33 \\
\hline 46 & \multicolumn{5}{|c|}{ Mexican IPC index } \\
\hline 47 & \multicolumn{5}{|c|}{ S\&P BSE Sensex } \\
\hline 48 & REI & $\begin{array}{c}\text { Reinet Investments } \\
\text { S.C.A. } \\
\end{array}$ & Speciality finance & 37130873697 & 39 \\
\hline 49 & TBS & Tiger Brands Limited & Consumer products & 59660806282 & 23 \\
\hline 50 & DSY & Discovery Limited & Insurance & 53002172524 & 28 \\
\hline 51 & REM & Remgro Limited & Consumer products & 88975812067 & 20 \\
\hline
\end{tabular}

